02

\title{
Влияние температуры на спектральные зависимости оптических свойств алюминия
}

\author{
() А.В. Каленский ${ }^{1}$, А.А. Звеков ${ }^{1,2}$, Б.П. Адуев ${ }^{2}$ \\ ${ }^{1}$ Кемеровский государственный университет, \\ 650000 Кемерово, Россия \\ ${ }^{2}$ Федеральный исследовательский центр угля и углехимии Сибирского отделения РАН \\ (Институт углехимии и химического материаловедения), \\ 650000 Кемерово, Россия \\ e-mail: kriger@kemsu.ru
}

Поступила в Редакцию 13.01.2017 г.

Проанализированы литературные данные по оптическим свойствам алюминия в области 198-1173 К. Предложены и апробированы аналитические выражения для описания зависимостей диэлектрической проницаемости алюминия от энергии кванта и температуры. Рассчитаны спектральные зависимости показателя поглощения и коэффициента зеркального отражения света алюминием при нормальном падении и коэффициентов эффективности поглощения света наночастицами алюминия в матрице фторида лития при различных температурах. Из полученных результатов следует появление необычного типа нелинейных эффектов поглощения света нанокомпозитами, содержащими наночастицы алюминия, проявляющихся в уменьшении коэффициента эффективности поглощения при увеличении температуры при энергиях кванта более $1.40 \mathrm{eV}$.

DOI: $10.21883 /$ OS.2018.04.45748.290-17

\section{Введение}

Температурные зависимости оптических свойств металлов и металлических наночастиц могут проявляться в ряде процессов, включающих абляцию, нелинейные оптические эффекты и инициирование химических реакций. При получении наночастиц абляцией металлов температурная зависимость коэффициента отражения приводит к корректировке значения критической плотности энергии импульса [1] и, следовательно, технологических характеристик процесса. В [2,3] показано, что изменение оптических свойств пленки молибдена при лазерном воздействии из-за нагревания и начала окисления приводит к уменьшению зоны абляции. Нагрев наночастиц с изменением их оптических свойств может вызывать нелинейные оптические эффекты в поле лазерного излучения [4]. В работах [5-7] сформулирована модель инициирования реакции взрывного разложения энергетического материала в окрестностях нагреваемой лазерным излучением металлической наночастицы без учета зависимости оптических свойств включения от температуры $(T)$. В то же время экспериментальное исследование температурных зависимостей оптических свойств массивных металлов и их наночастиц представляет собой нетривиальную задачу. Для большинства металлов имеются только отрывочные сведения, касающиеся влияния температуры на коэффициент отражения при определенной длине волны, которых недостаточно для прогнозирования оптических свойств наночастиц при различных температурах. Цель работы: на основе анализа литературных данных получить аппроксимирующие выра- жения для диэлектрической проницаемости алюминия при различных температурах и энергиях кванта света, рассчитать спектральные зависимости показателя поглощения, коэффициентов отражения металла и эффективности поглощения наночастиц алюминия в области $200-1100 \mathrm{~K}$.

Алюминий выбран в качестве объекта исследования по следующим причинам: для него имеются экспериментальные оптические константы в широком температурном и спектральном диапазонах $[8,9]$; он является модельным объектом при исследовании процессов абляции [10]; наночастицы алюминия используются в качестве добавки к взрывчатым веществам и порохам для повышения их чувствительности к лазерному излучению $[7,11,12]$. В двух последних примерах оптические свойства металла играют определяющую $[13,14]$ роль, и их учет необходим при формулировке моделей данных процессов.

\section{Спектрально-температурные зависимости оптических констант алюминия}

Для прогнозирования влияния температуры на оптические свойства наночастиц алюминия необходимо получить спектрально-температурные зависимости оптических констант. Экспериментальные измерения показателя поглощения света напыленными пленками алюминия в вакууме при температурах 198, 298, 404 и $552 \mathrm{~K}$ выполнены в [8] в области энергий кванта $(E)$ от 0.64 до $2.5 \mathrm{eV}$. Результаты измерений приведены на 

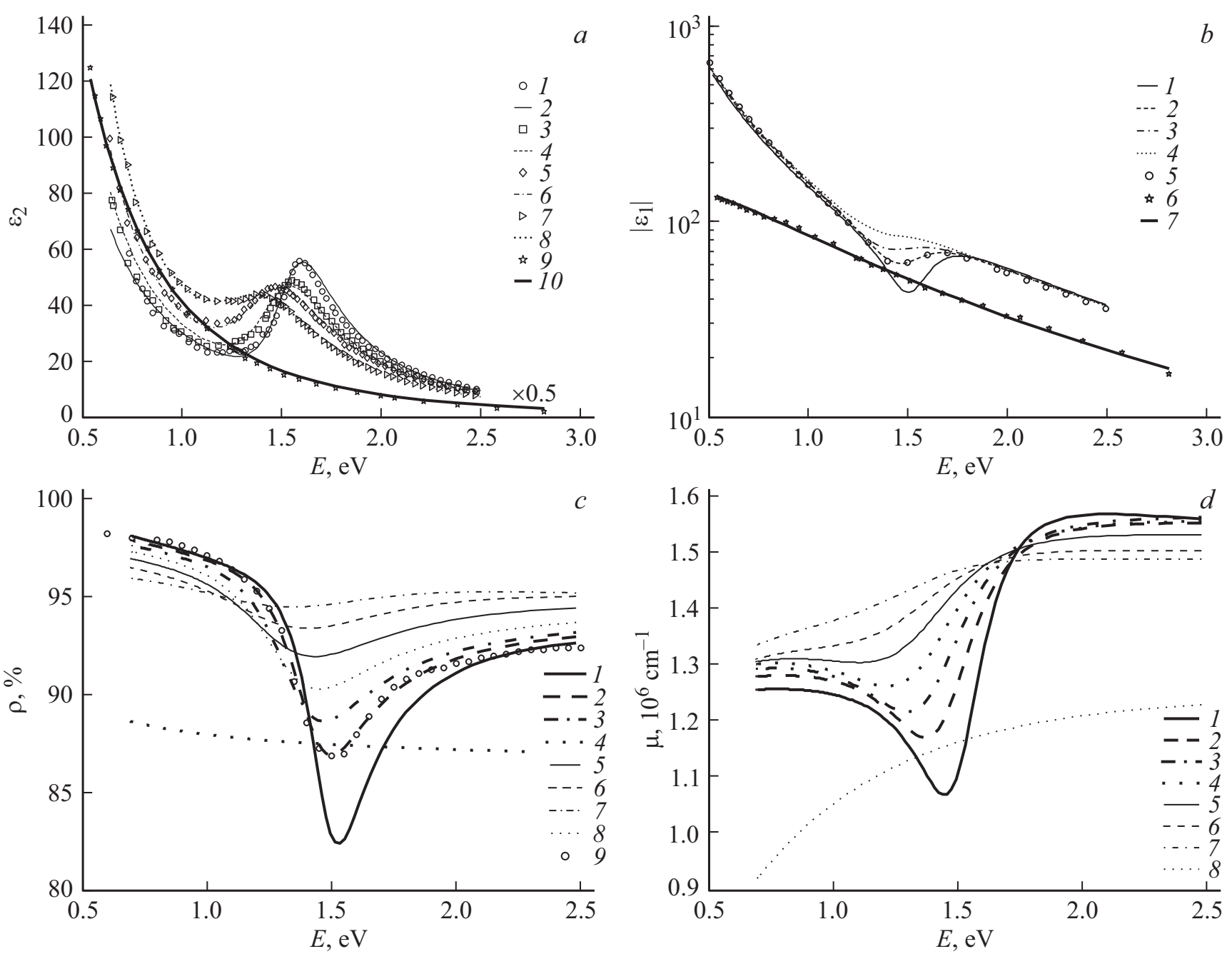

Рис. 1. Спектральные зависимости мнимой $(a)$ и действительной $(b)$ частей диэлектрической проницаемости, коэффициентов отражения при нормальном падении $(c)$ и показателя поглощения $(d)$ алюминия при различных температурах. Обозначения в тексте.

рис. 1, $a$ в виде спектральных зависимостей мнимой части диэлектрической проницаемости $\varepsilon_{2}(E)$ при температурах 198 (кривая 1), 298 (3), 404 (5) и $552 \mathrm{~K}$ (7). На зависимости $\varepsilon_{2}(E)$ хорошо проявляется полоса с локальным максимумом в области $1.5 \mathrm{eV}$, форма которой отличается от лоренцевой [8]. Данная полоса связана с межзонным поглощением, и ее интерпретации были посвящены работы $[15,16]$. В $[1,17]$ предложено использовать модель критических точек для описания оптических констант алюминия. В то же время аппроксимация экспериментальных данных выполнена только в видимой области без анализа влияния температуры на получаемые параметры [17]. Возрастание мнимой части диэлектрической проницаемости при уменьшении энергии кванта в области менее $1 \mathrm{eV}$ связано не только с внутризонным поглощением света, но и со слабой полосой межзонного поглощения с максимумом в районе $0.5 \mathrm{eV}$. В настоящей работе мы использовали следующее выражение для аппроксимации зависимости
$\varepsilon_{2}(E)$ в области $0.64-2.5 \mathrm{eV}$ :

$$
\varepsilon_{2}(E)=A_{1} E^{A_{2}}+\frac{E^{A_{4}}}{A_{3}}\left[1+\exp \left(-\frac{E-A_{6}}{A_{5}}\right)\right],
$$

где энергия кванта нормирована на $1 \mathrm{eV}$. Параметры уравнения (1) вычислялись для каждой из 4-х температур. После этого температурная зависимость параметров $A$ аппроксимировалась квадратным трехчленом

$$
A_{i}=a_{i 0}+a_{i 1}(T / 300)+a_{i 2}(T / 300)^{2},
$$

где температура (в K) нормирована на комнатную температуру $(300 \mathrm{~K})$. Результаты аппроксимации по выражению (1) приведены на рис. 1, $a$ при температурах 198 (2), 298 (4), 404 (6) и 552 K (8) при значениях параметров, представленных в таблице. Из таблицы следует, что все параметры, кроме $A_{3}$, имеют сильную температурную зависимость, которая при этом существенно нелинейная. 
Параметры аппроксимации спектральных зависимостей мнимых частей диэлектрической проницаемости алюминия при различных температурах в спектральном диапазоне $0.64-2.5 \mathrm{eV}$

\begin{tabular}{c|c|c|c|c|c|c}
\hline$a$ & $A_{1}$ & \multicolumn{1}{|c|}{$A_{2}$} & $A_{3}$ & \multicolumn{1}{c|}{$A_{4}$} & \multicolumn{1}{c}{$A_{5}$} & $A_{6}$ \\
\hline$a_{i 0}$ & 25.6829 & 1.2711 & -2.2597 & -9.2965 & $3.3477 \cdot 10^{-2}$ & 1.7026 \\
$a_{i 1}$ & 5.9058 & -0.9069 & $5.5674 \cdot 10^{-3}$ & 5.9985 & $3.4032 \cdot 10^{-2}$ & -0.2834 \\
$a_{i 2}$ & 1.1469 & 0.1665 & $-1.0550 \cdot 10^{-3}$ & -1.9412 & $2.9111 \cdot 10^{-3}$ & $5.6784 \cdot 10^{-2}$
\end{tabular}

Для расчета действительной части диэлектрической проницаемости использовались соотношения КрамерсаКронига. При этом для функции $\varepsilon_{2}(E)$ использовалось выражение (1) с параметрами (таблица) при энергии кванта $E \geq 0.45 \mathrm{eV}$. В области $E<0.45 \mathrm{eV}$ использовались выражения, следующие из теории Друде:

$$
\varepsilon_{1 D}(E)=1-\frac{E_{p}^{2}}{\left(E^{2}+\Gamma^{2}\right)}, \quad \varepsilon_{2 D}(E)=\frac{\Gamma E_{0}^{2}}{E\left(E^{2}+\Gamma^{2}\right)},
$$

где $E_{p}=\hbar \sqrt{\frac{4 \pi n_{e} e^{2}}{m_{e}}}-$ энергия плазменных колебаний, $\Gamma=\frac{n_{e} e^{2}}{m_{e f} \sigma_{0}}-$ релаксационный параметр, выраженный в единицах энергии, $e-$ заряд электрона, $m_{e}-$ масса покоя электрона, $n_{e}$ - объемная концентрация валентных электронов, $m_{e f}$ - эффективная масса электрона в металле, $\sigma_{0}-$ статическая проводимость. Концентрация валентных электронов определялась по формуле

$$
n_{e}=\eta \frac{N_{A} d}{M}
$$

где $\eta$ - количество валентных электронов в атоме, $N_{A}$ - число Авогадро, $M$ - атомная масса, $d-$ плотность.

Результаты расчетов $\varepsilon_{1}(E)$ с использованием выражений (1), (2) приведены по модулю на рис. 1, $b$ при температурах 198 (1), 298 (2), 404 (3) и 552 K (4). Одновременно на рис. $1, b$ показана экспериментальная зависимость при комнатной температуре (5) [18]. На рисунке хорошо выделяется локальный минимум при энергии кванта около $1.5 \mathrm{eV}$, соответствующий полосе межзонного поглощения. Как и на зависимости $\varepsilon_{2}(E)$, интенсивность полосы уменьшается с ростом $T$. Вдали от межзонной полосы температура незначительно влияет на величину действительной части диэлектрической проницаемости.

При температурах выше точки плавления межзонные полосы на спектральных зависимостях диэлектрической проницаемости исчезают [9], что позволяет использовать выражения из теории Друде (2). Результаты аппроксимации формулами (2) результатов измерений [9] при $T=1173 \mathrm{~K}$ (рис. 1) приводят к величинам $E_{p}=12.84 \mathrm{eV}$ и $\Gamma=0.964 \mathrm{eV}$. При расчетах использован температурный коэффициент сопротивления расплавленного алюминия $1.66 \cdot 10^{-8} \Omega \cdot \mathrm{cm} \cdot \mathrm{K}^{-1}[19]$. На рис. $1, a(9,10)$ представлены экспериментальная [9] и рассчитанная по уравнению (2) спектральные зависимости мнимой части диэлектрической проницаемости расплава алюминия при $T=1173$. На рис. $1, b$ показана экспериментальная [9] зависимость $\varepsilon_{1}(E)$ расплава алюминия при температуре $1173 \mathrm{~K}$ и ее аппроксимация по уравнению (2). Эффективная масса электрона для расплавленного алюминия была выбрана равной $m_{e f}=1.28 m_{e}$ для оптимального описания экспериментальных данных при $T=1173 \mathrm{~K}[9]$.

На рис. 1, $с$ приведены рассчитанные при температурах 200 (1), 300 (2), 400 (3), 500 (4), 600 (5), $700(6), 800$ (7) и $1000 \mathrm{~K}(8)$ спектральные зависимости коэффициента отражения $(\rho)$ алюминия при нормальном падении света, а также экспериментальная зависимость $\rho(E)$ при $T=298 \mathrm{~K}$ [18]. Совпадение рассчитанной зависимости при $300 \mathrm{~K}$ с экспериментальной находится в пределах 0.5\%. По мере повышения температуры в области больших энергий кванта происходит рост коэффициента отражения вплоть до температуры плавления. Обратная тенденция имеет место при малых энергиях кванта. Наибольшие изменения видны в области полосы межзонного поглощения, например, при энергии кванта $1.5 \mathrm{eV}$ коэффициент отражения возрастает от 82.7 до $94.5 \%$ при увеличении температуры от 200 до $800 \mathrm{~K}$. В спектральной области $0.90-1.43 \mathrm{eV}$ температурная зависимость коэффициента отражения ниже точки плавления немонотонна. Расплавление металла приводит к исчезновению межзонных полос и слабой зависимости коэффициента отражения от энергии кванта падающего света.

На рис. $1, d$ представлены рассчитанные спектральные зависимости показателя поглощения алюминия при температурах 200 (1), 300 (2), 400 (3), 500 (4), 600 (5), $700(6), 800$ (7) и $1000 \mathrm{~K}(8)$. Минимум показателя поглощения при $T=200 \mathrm{~K}$ наблюдается при энергии кванта $1.45 \mathrm{eV}$. При увеличении температуры происходит его батохромный сдвиг с уменьшением значения в точке минимума. В результате величина показателя поглощения возрастает в данной области в 1.35 раза при увеличении температуры от 200 до $900 \mathrm{~K}$. В области малых энергий кванта рост температуры приводит к увеличению показателя поглощения, а в области больших $(E>1.8 \mathrm{eV})$ преобладает тенденция к уменьшению показателя преломления. При $T=1000 \mathrm{~K}$ благодаря расплавлению вещества полоса исчезает, и показатель поглощения монотонно увеличивается с ростом энергии кванта. 

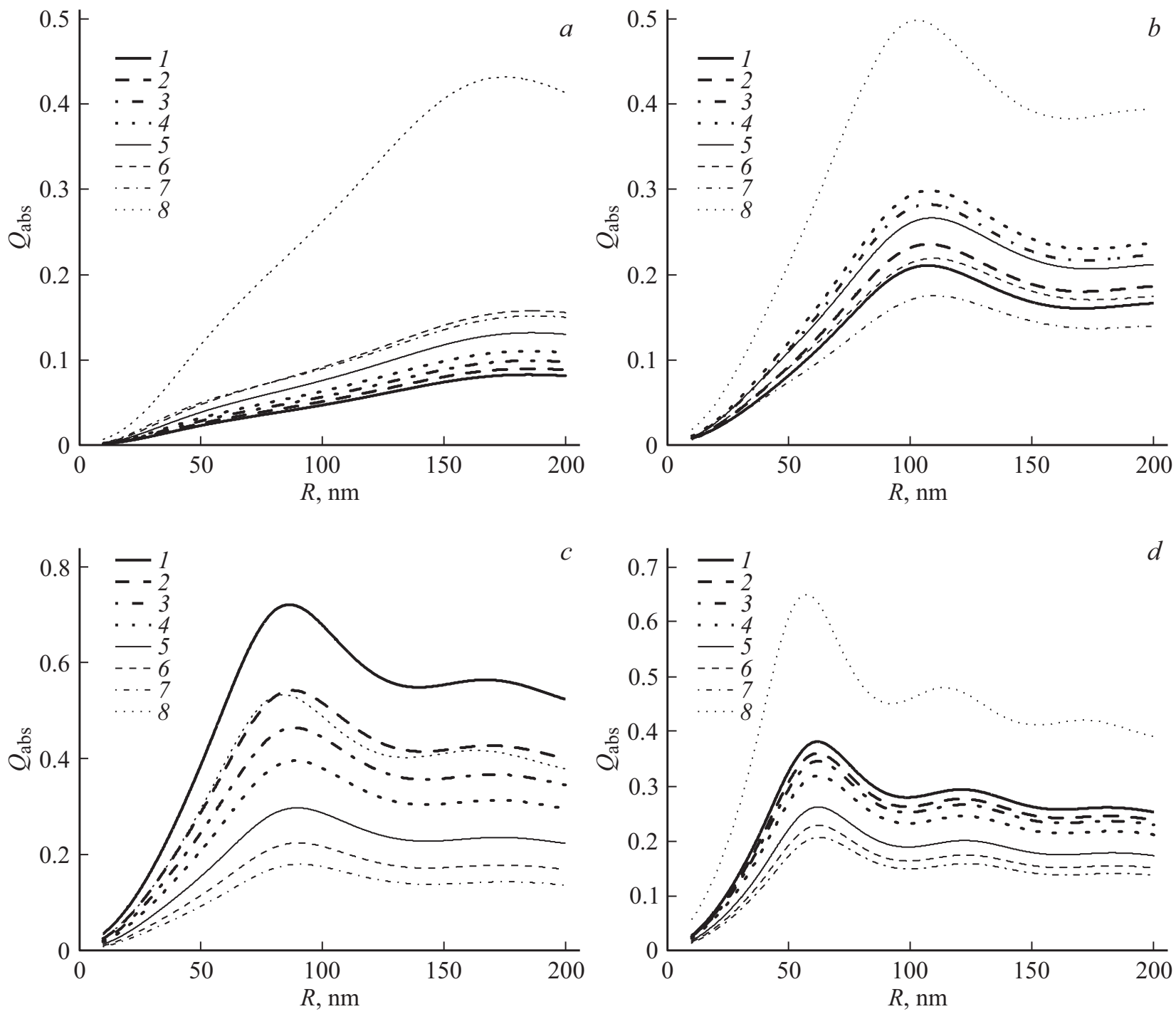

Рис. 2. Зависимости коэффициентов эффективности поглощения от радиуса наночастиц алюминия при энергиях кванта $0.75(a)$, $1.25(b), 1.5(c)$ и $2.1(d)$ и температурах $200(1), 300(2), 400(3), 500(4), 650(5), 800(6), 900(7)$ и $1000 \mathrm{~K}(8)$ в матрице LiF.

\section{Влияние температуры на оптические свойства наночастиц Al}

Рассмотрим влияние температуры на оптические свойства наночастиц алюминия в матрице фторида лития, для которого известны спектральные зависимости показателя преломления и его температурного коэффициента [20]. На рис. 2 представлены рассчитанные зависимости коэффициента эффективности поглощения наночастиц алюминия от их радиуса $(R)$ при различных значениях температуры и энергиях кванта $0.75(a)$, $1.25(b), 1.5(c)$ и $2.1 \mathrm{eV}(d)$. Значительного влияния температуры на величину коэффициента эффективности рассеяния выявлено не было, что согласуется с нашими результатами, полученными для наночастиц серебра [4]. При наименьшей из использованных энергий кванта $0.75 \mathrm{eV}$ увеличение температуры приводит к росту коэффициента эффективности поглощения. При $T=200 \mathrm{~K}$ максимальный коэффициент эффективности поглощения, равный 0.0826 , имеют наночастицы с радиусом $185.0 \mathrm{~nm}$, при увеличении температуры до $800 \mathrm{~K}$ амплитуда максимума возрастает до 0.1574 с его незначительным сдвигом вдоль оси абсцисс до $186.5 \mathrm{~nm}$. Далее следует небольшое уменьшение амплитуды максимума при температуре от $900 \mathrm{~K}$ до 0.1516. Плавление с увеличением температуры до $1000 \mathrm{~K}$ приводит к смещению радиуса в максимуме к $175.2 \mathrm{~nm}$ с увеличением амплитуды до 0.4319. При энергии кванта $1.25 \mathrm{eV}$ рост температуры от 200 до $900 \mathrm{~K}$ приводит к увеличению абсциссы максимума с 107.3 до $109.6 \mathrm{~nm}$. Амплитуда максимума вначале растет до температуры $490 \mathrm{~K}$, затем начинается ее уменьшение вплоть до температуры плавления, при превышении которой происходит рост амплитуды максимума до 0.4984. Увеличение энергии кванта до $1.5 \mathrm{eV}$ делает зависимость коэффициента эффективности поглощения от температуры, монотонно 
уменьшающейся при температурах ниже точки плавления. В частности, амплитуда максимума уменьшается от 0.7206 при температуре $200 \mathrm{~K}$ до 0.2240 при $900 \mathrm{~K}$. В отличие от результатов при меньших значениях энергии кванта коэффициент эффективности поглощения при температуре $1000 \mathrm{~K}$ ниже, чем при $200 \mathrm{~K}$, а зависимости, рассчитанные при температурах 300 и $1000 \mathrm{~K}$, почти совпадают. При энергии кванта $2.1 \mathrm{eV}$ ниже точки плавления сохраняется тенденция к уменьшению $Q_{\mathrm{abs}}$ при росте температуры. Амплитуда основного максимума уменьшается в 1.67 раза при увеличении температуры от 200 до $900 \mathrm{~K}$. При плавлении вещества и повышении температуры до $1000 \mathrm{~K}$ амплитуда максимума превышает значение при $200 \mathrm{~K}$ в 1.70 раз. Рассмотрение влияния температуры на коэффициент эффективности поглощения при фиксированной энергии кванта позволило сделать вывод, что при $E<1.40 \mathrm{eV}$ наблюдаются немонотонные зависимости $Q_{\mathrm{abs}}(T)$. Температура, при которой величина коэффициента эффективности поглощения максимальна, возрастает при уменьшении энергии кванта. Энергия кванта, при которой происходит смена характера зависимости $(1.40 \mathrm{eV})$, практически не зависит от радиуса наночастиц алюминия.

На рис. 3 приведены рассчитанные спектральные зависимости коэффициентов эффективности поглощения света наночастицами алюминия с радиусами 30 (a), 50 (b), 80 (c) и $120 \mathrm{~nm} \mathrm{(d)} \mathrm{при} \mathrm{температурах} \mathrm{в}$ интервале $200-1000 \mathrm{~K}$. На спектральных зависимостях выделяется полоса поглощения, интенсивность которой уменьшается при росте температуры. В результате полоса вначале превращается в плечо и затем исчезает при высоких температурах. При температуре $1000 \mathrm{~K}$ (выше точки плавления) на спектральной зависимости $Q_{\mathrm{abs}}$ полоса поглощения не выделяется. Из полученных спектральных зависимостей следует, что температура оказывает противоположное влияние в области малых и больших энергий кванта. Например, при росте температуры от $200 \mathrm{~K}$ до температуры плавления для наночастиц с радиусом $10 \mathrm{~nm}$ коэффициент эффективности поглощения при энергии кванта $0.7 \mathrm{eV}$ возрастает от $1.10 \cdot 10^{-3}$ до $2.61 \cdot 10^{-3}$, тогда как при $E=2.4 \mathrm{eV}$ уменьшается от $3.413 \cdot 10^{-2}$ до $1.854 \cdot 10^{-2}$. В точке плавления происходит рост $Q_{\mathrm{abs}}$ при энергии кванта 0.7 и $2.4 \mathrm{eV}$ до $5.80 \cdot 10^{-3}$ и $7.931 \cdot 10^{-2}$ соответственно, а при дальнейшем увеличении температуры до $1100 \mathrm{~K}$ наблюдается увеличение до $6.09 \cdot 10^{-3}$ и $8.531 \cdot 10^{-2}$. В случае наночастиц с радиусом $100 \mathrm{~nm}$ в интервале температур $200 \mathrm{~K}$-точка плавления происходит увеличение коэффициента эффективности поглощения в 2.31 раза при энергии кванта $0.7 \mathrm{eV}$ и уменьшение в 1.77 раза при $E=2.4 \mathrm{eV}$. Таким образом, относительное изменение $Q_{\text {abs }}$ при росте температуры вдали от полосы поглощения слабо зависит от радиуса наночастиц.

Наиболее заметное влияние на величину коэффициента эффективности поглощения температура оказывает в области полосы поглощения. На рис. 4 показаны рассчитанные температурные зависимости энергии кванта в максимуме полосы $(a)$, соответствующего значения коэффициента эффективности поглощения $Q_{m}(b)$ и полуширины полосы $(c)$ в области малых энергий кванта. Для вычисления полуширины спектр в коротковолновой и длинноволновой областях вне полосы поглощения аппроксимировался рядом Лорана с сохранением 5 членов разложения, что обеспечивало „базовую линию“. Полувысота определялась как половина разности $Q_{m}$ и соответствующего уровня „базовой линии“. Расчет положения максимума полосы прекращали при его превращении в плечо. Для наночастиц малого радиуса в температурном диапазоне 200-300 K положение максимума на оси энергий кванта практически не изменяется, затем наблюдается гипсохромный сдвиг (рис. 4, $a$ ). Одновременно видно снижение амплитуды максимума, так что в области появления гипсохромного сдвига отличия максимума от плеча становятся неразличимыми. Для наночастиц с радиусом более $100 \mathrm{~nm}$ прослеживается батохромный сдвиг при росте температуры. Например, в случае наночастиц радиуса $120 \mathrm{~nm}$ максимум полосы сдвигается вдоль оси абсцисс от 1.516 до $1.308 \mathrm{eV}$ при увеличении температуры от 200 до $700 \mathrm{~K}$. Для наночастиц всех радиусов происходит уменьшение амплитуды максимума коэффициента эффективности поглощения $Q_{m}$ по мере роста температуры (рис. 4,b). В частности, в интервале $200-400 \mathrm{~K}$ амплитуда максимума уменьшается в 1.66 раза для наночастиц радиуса $(R)$ $10 \mathrm{~nm}$, в 1.58 раз - при $R=30 \mathrm{~nm}$, в 1.62 раза при $R=80 \mathrm{~nm}$ и в 1.51 раз - при $R=100 \mathrm{~nm}$. То есть относительное изменение амплитуды максимума полосы поглощения наночастиц слабо зависит от их радиуса. Также независимо от радиуса наночастиц полуширина полосы поглощения растет по мере увеличения температуры. Произведение амплитуды максимума полосы поглощения на ее полуширину изменяется в пределах 10\% в области температур $200-400 \mathrm{~K}$ для наночастиц с радиусами $30-70 \mathrm{~nm}$. Для наночастиц большего радиуса температурная область практического постоянства произведения $Q_{m} \Delta E$ значительно расширяется.

При превышении температуры плавления на спектральных зависимостях $Q_{\text {abs }}$ возникает максимум, лежащий для наночастиц с радиусом менее $50 \mathrm{~nm}$ в области энергий кванта больших, чем $2.5 \mathrm{eV}$. Энергия кванта в точке максимума изменяется обратно пропорционально радиусу наночастицы. Данная зависимость отмечалась нами ранее в [21]. Для наночастиц с $R>100 \mathrm{~nm} \mathrm{в}$ синей части рассматриваемого спектрального диапазона появляется также второй максимум. Наибольший относительный рост коэффициента эффективности поглощения (в 4.3 раза) при пересечении точки плавления наблюдается для наночастиц с радиусом менее $50 \mathrm{~nm}$ в синей области спектра. Относительное увеличение $Q_{\mathrm{abs}}$ в красной области спектра для малых наночастиц меньше, чем в синей. При увеличении радиуса наночастиц относительное увеличение $Q_{\mathrm{abs}}$ начинает меньше зависеть от энергии кванта, находясь в области 3-3.5 раз. 

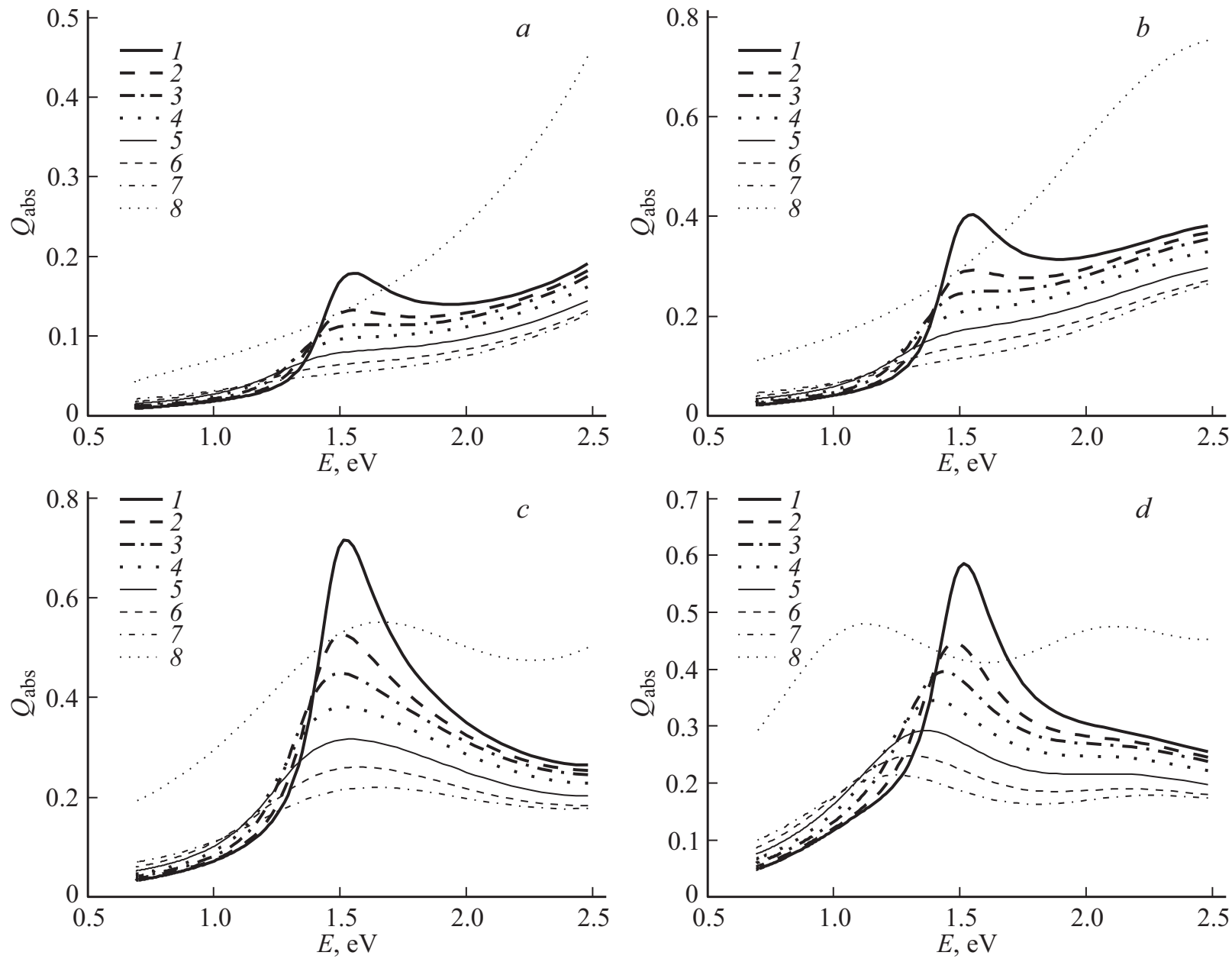

Рис. 3. Спектральные зависимости коэффициентов эффективности поглощения наночастиц алюминия с радиусами $30(a), 50(b)$, $80(c)$ и $120(d)$ и при температуре $200(1), 300(2), 400(3), 500(4), 650(5), 800(6), 900(7)$ и $1000 \mathrm{~K}(8)$ в матрице LiF.

\section{Обсуждение результатов}

Особенность результатов расчетов заключается в том, что при энергиях кванта более $1.40 \mathrm{eV}$ увеличение температуры приводит к уменьшению коэффициента эффективности поглощения. Данное поведение является аномальным для металлов, поскольку рост температуры обычно приводит к увеличению скорости диссипации энергии и понижению коэффициента отражения. В случае наночастиц это должно приводить к росту $Q_{\mathrm{abs}}$ [4]. Для наночастиц алюминия в области энергий кванта порядка $1.5 \mathrm{eV}$ основной вклад в оптические свойства вносит полоса межзонного поглощения, положение и интенсивность которой чувствительны к температуре. При увеличении $T$ интенсивность полосы уменьшается вместе с увеличением ее характерной ширины и батохромным сдвигом. Это приводит к соответствующему сдвигу минимума на зависимости $\rho(E)$ и максимума на зависимости $Q_{\text {abs }}(E)$. Также следует отметить, что максимум полосы поглощения в спектрах наночастиц (рис. 3) близок к максимуму полосы межзонного поглощения массивного металла (рис. $1, a$ ).
Изменение характера зависимости коэффициента отражения от температуры, наблюдаемое при варьировании длины волны, должно проявляться в экспериментах по лазерной абляции алюминиевых мишеней. Так как показатель поглощения алюминия достаточно большой (рис. $1, d)$, коэффициент поглощения мишени равен $1-\rho$. При энергии кванта $1 \mathrm{eV}$ он увеличивается в 1.51 раза при увеличении температуры от 300 до $800 \mathrm{~K}$, при $E=1.5 \mathrm{eV}$, наоборот, уменьшается в 2.4 раза, а при $E=2.3 \mathrm{eV}$ уменьшается в 1.52 раза.

Влияние температуры на коэффициент эффективности поглощения света наночастицами может приводить к нелинейным эффектам при их лазерном нагреве, который будет проявляться как изменение коэффициента пропускания композита на основе прозрачной матрицы при увеличении плотности энергии импульса. В отличие от изучаемых в последнее время нелинейных эффектов в системах, содержащих наночастицы металлов, рассматриваемый случай имеет ряд особенностей: эффект проявляется для достаточно массивных наночастиц (радиусом порядка $50 \mathrm{~nm}$ или более), тогда как большинство работ выполнены с наночастицами благородных метал- 

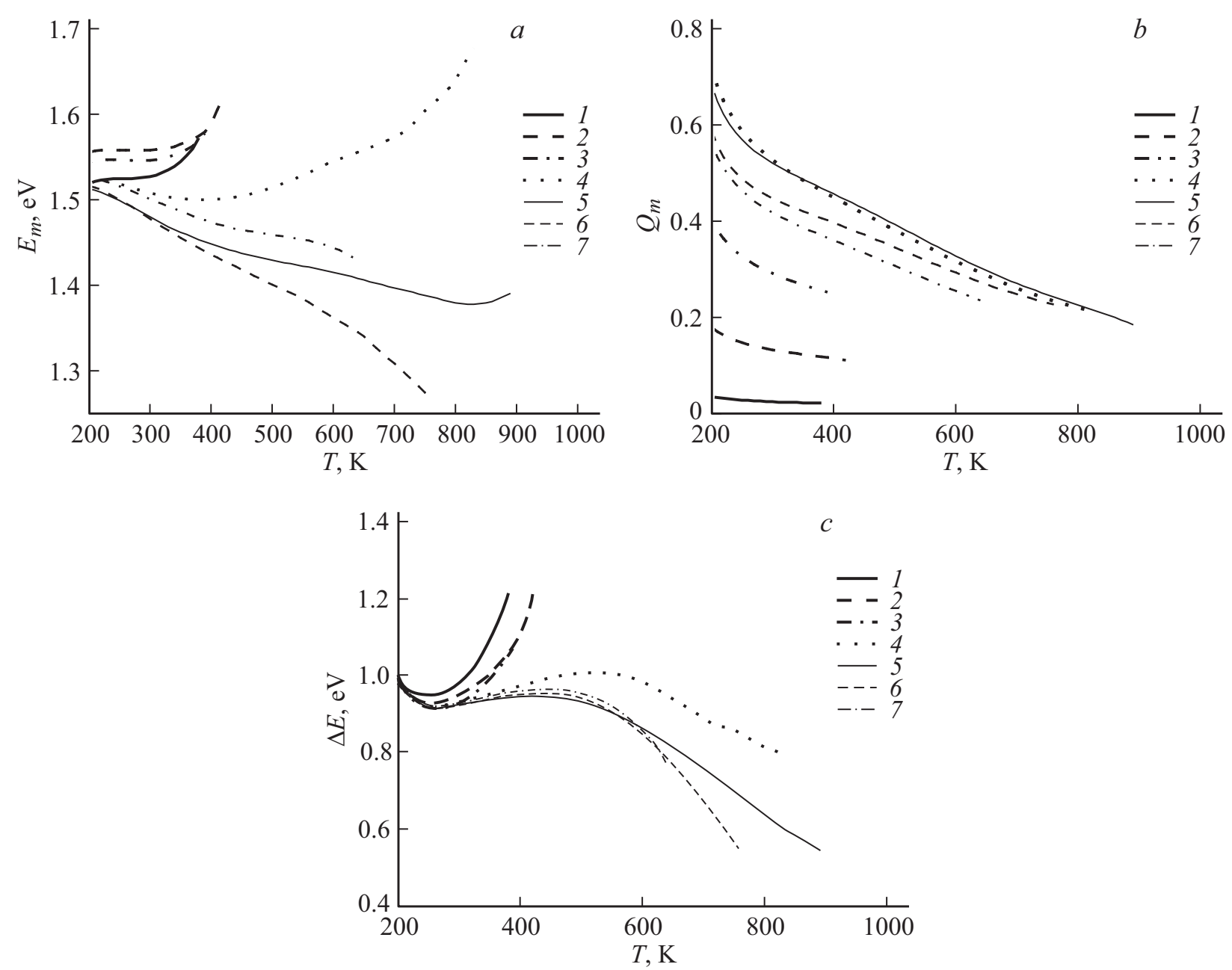

Рис. 4. Температурные зависимости энергии кванта в максимуме $(a)$, амплитуды $(b)$ и полуширины $(c)$ полосы поглощения на спектральных зависимостях коэффициента эффективности поглощения наночастиц алюминия, имеющих радиус 10 (1), 30 (2), $50(3), 80(4), 100(5), 120$ (6) и $150 \mathrm{~nm}$ (7) в матрице LiF.

лов радиусами порядка $10 \mathrm{~nm}$ [22-24]; расчеты проведены для одиночных наночастиц, поэтому эффекты могут проявляться и в области малых концентраций металла, где взаимодействием наночастиц можно пренебречь [25]; наибольшая температура наночастиц достигается при длительностях импульса, сопоставимых с характерным временем теплоотвода в матрицу [26], поэтому для наночастиц радиуса $50 \mathrm{~nm}$ наиболее эффективно данные процессы будут инициироваться импульсами с длительностью порядка $10 \mathrm{~ns}$. Как увеличение, так и уменьшение длительности импульса с сохранением плотности энергии будет приводить к меньшей выраженности нелинейного поглощения света образцом.

В цикле работ [4-7,14,26] рассмотрен процесс нагревания наночастиц металлов импульсным лазерным излучением и связанных с ним процессов плавления наночастиц и матрицы, инициирования реакции разложения матрицы. Величина критической плотности энергии лазерного инициирования композитов взрывчатое вещество-наночастицы металлов определяется оп- тическими [6], теплофизическими [5,26] и механическими [27] свойствами системы. Особую роль играют нелинейные эффекты, способные привести к значительной коррекции предсказаний модели. Включение в модель учета нелинейного взаимодействия света с наночастицами, которое связано с изменением коэффициента эффективности поглощения при росте температуры, является необходимым шагом ее развития.

\section{Заключение}

В работе проанализированы литературные данные по спектральным зависимостям оптических свойств алюминия в области температур 198-1173 К. Предложены и апробированы приближенные выражения для описания зависимостей диэлектрической проницаемости алюминия от энергии кванта и температуры. Рассчитаны спектральные зависимости показателя поглощения и коэффициента зеркального отражения света алюминием при нормальном падении и коэффициентов эффективности 
поглощения света наночастицами алюминия в матрице фторида лития при различных температурах. Показано, что при энергии кванта менее $1.4 \mathrm{eV}$ наблюдается немонотонная зависимость коэффициента эффективности поглощения света наночастицами от температуры (возрастание, затем убывание), коррелирующая с коэффициентом поглощения металла. Из полученных результатов следует появление необычного типа нелинейных эффектов поглощения света нанокомпозитами, содержащими наночастицы алюминия.

Работа выполнена при финансовой поддержке Министерства образования и науки РФ (задание № 3.5363.2017/8.9).

\section{Список литературы}

[1] Marla D., Bhandarkar U.V., Joshi S.S. // J. Phys. D. 2014. V. 47. N 10. P. 105306. doi 10.1088/0022-3727/47/10/105306

[2] Казанский Н.Л., Полетаев С.Д. // ЖТФ. 2016. Т. 86. № 9. C. 1; Kazanskiy N.L., Poletayev S.D. // Tech. Phys. 2016. V. 61. N 9. P. 1279. doi 10.1134/S1063784216090127

[3] Волков А.В., Казанский Н.Л., Моисеев О.Ю., Паранин В.Д., Полетаев С.Д., Чистяков И.В. // ЖТФ. 2016. Т. 86. № 4. C. 101; Volkov A.V., Kazanskiy N.L., Moiseev O.Yu., Paranin V.D., Poletayev S.D., Chistyakov I.V. // Tech. Phys. 2016. V. 61. N 4. P. 579. doi $10.1134 / \mathrm{S} 1063784216040241$

[4] Каленский А.В., Звеков А.А., Никитин А.П. // Журн. прикл. спектр. 2016. Т. 83. № 6. С. 972; Kalenskii A.V., Zvekov A.A., Nikitin A.P. // J. Appl. Spectr. 2017. V. 83. N 6. P. 972. doi 10.1007/s10812-017-0400-z

[5] Александров Е.И., Ципилев В.П. // Физика горения и взрыва. 1984. Т. 20. № 6. С. 104; Alexandrov E.I., Tsipilev V.P. // Combust. Expl. Shock Waves. 1984. V. 20. N 6. P. 690. doi 10.1007/BF00757322

[6] Каленский А.В., Ананьева М.В., Звеков А.А., Зыков И.Ю. // Физика горения и взрыва. 2016. Т. 52. № 2. С. 122; Kalenskii A.V., Anan'eva M.V., Zvekov A.A., Zykov I.Yu. // Combust. Expl. Shock Waves. 2016. V. 52. N 2. P. 234. doi 10.1134/S0010508216020143

[7] Каленский А.В., Ананьева М.В., Звеков А.А., Зыков И.Ю. // ЖТФ. 2015. Т. 85. № 3. C. 119; Kalenskii A.V., Anan'eva M.V., Zvekov A.A., Zykov I.Yu. // Tech. Phys. 2015. V. 60. N 3. P. 437. doi 10.1134/S1063784215030081

[8] Mathewson A.G., Myers H.P. // J. Phys. F. 1972. V. 2. N 2. P. 403. doi 10.1088/0305-4608/2/2/030

[9] Акашев Л.А., Кононенко В.И. // ТВТ. 2001. Т. 39. № 3. C. 412; Akashev L.A., Kononenko V.I. // High Temperature. 2001. V. 39. N 3. P. 384. doi 10.1023/A:1017502424054

[10] Бежсанов С.Г., Канавин А.П., Урюпин С.А. // Квант. электрон. 2016. T. 46. № 2. C. 119; Bezhanov S.G., Kanavin A.P., Uryupin S.A. // Quantum Electron. 2016. V. 46. N 2. P. 119. doi 10.1070/QEL15877

[11] Архипов В.А., Коротких А.Г., Кузнецов В.Т., Раздобреев А.А., Евсеенко И.А. // Хим. физика. 2011. Т. 30. № 7. C. 68; Arkhipov V.A., Korotkikh A.G., Kuznetsov V.T., Razdobreev A.A., Evseenko I.A. // Russ. J. Phys. Chem. B. 2011. V. 5. N 4. P. 616. doi 10.1134/S1990793111040026
[12] Адуев Б.П., Нурмухаметов Д.Р., Фурега Р.И., Звеков А.А., Каленский А.В. // Хим. физика. 2013. Т. 32. № 8. С. 39; Aduev B.P., Nurmukhametov D.R., Furega R.I., Zvekov A.A., Kalenskii A.V. // Russ. J. Phys. Chem. B. 2013. V. 7. N 4. P. 453. doi 10.1134/S199079311304012X

[13] Кригер В.Г., Каленский А.В., Звеков А.А., Зыков И.Ю., Адуев Б.П. // Физика горения и взрыва. 2012. Т. 48. № 6. C. 54; Kriger V.G., Kalenskii A.V., Zvekov A.A., Zykov I.Yu., Aduev B.P. // Combust. Expl. Shock Waves. 2012. V. 48. N 6. P. 705. doi 10.1134/S001050821206007X

[14] Каленский А.В., Звеков А.А., Ананьева М.В., Зыков И.Ю., Кригер В.Г., Адуев Б.П. // Физика горения и взрыва. 2014. T. 50. № 3. C. 98; Kalenskii A.V., Zvekov A.A., Anan'eva M.V., Zykov I.Yu., Kriger V.G., Aduev B.P. // Combust. Expl. Shock Waves. 2014. V. 50. N 3. P. 333. doi 10.1134/S0010508214030113

[15] Ashcroft N.W., Sturm K. // Phys. Rev. B. 1971. V. 3. N 6. P. 1898. doi 10.1103/PhysRevB.3.1898

[16] Brust D. // Phys. Rev. B. 1970. V. 2. N 4. P. 818. doi 10.1103/PhysRevB.2.818

[17] Barchiesi D., Grosges Th. // J. Nanophotonics. 2014. V. 8. N 1. P. 083097. doi 10.1117/1.JNP.8.083097

[18] Palik E.D. Handbook of Optical Constants of Solids. San Diego: Academic Press Inc., 1985. V. 1. P. 405.

[19] Brandt R., Neuer G. // Int. J. Thermophys. 2007. V. 28. N 5. P. 1429. doi 10.1007/s10765-006-0144-0

[20] Li H.H. // J. Phys. Chem. Ref. Data. 1976. V. 5. N 2. P. 329. doi $10.1063 / 1.555536$

[21] Каленский А.В., Звеков А.А., Никитин А.П., Ананьева М.В., Адуев Б.П. // Опт. и спектр. 2015. Т. 118. № 6. C. 1012; Kalenskii A.V., Zvekov A.A., Nikitin A.P., Anan'eva M.V., Aduev B.P. // Opt. Spectrosc. 2015. V. 118. N 6. P. 978. doi 10.1134/S0030400X15060119

[22] Ghanipour M., Dorranian D. // Опт. и спектр. 2015. T. 118. № 6. C. 981; Ghanipour M., Dorranian D. // Opt. Spectrosc. 2015. V. 118. N 6. P. 949 . doi 10.1134/S0030400X15060132

[23] Ghambari T., Dorranian D. // Опт. спектр. 2015. T. 119. № 5. C. 816; Ghambari T., Dorranian D. // Opt. Spectrosc. 2015. V. 119. N 5. P. 838. doi 10.1134/S0030400X15110089

[24] Рассказов И.Л., Маркель В.А., Карпов С.В. // Опт. спектр. 2013. T. 115. № 5. C. 753; Rasskazov I.L., Markel V.A., Karpov S.V. // Opt. Spectrosc. 2013. V. 115. N 5. P. 666. doi 10.1134/S0030400X13110180

[25] Ряснянский A.И., Palpant B., Debrus S., Pal U., Степанов А.Л. // ФТТ. 2009. Т. 51. № 1. С. 52; Ryasnyanskiy A.I., Palpant B., Debrus S., Pal U., Stepanov A.L. // Phys. Solid State. 2009. V. 51. N 1. P. 55. doi 10.1134/S1063783409010065

[26] Кригер В.Г., Каленский А.В., Звеков А.А., Зыков И.Ю., Никитин А.П. // Теплофизика и аэромеханика. 2013. Т. 20. № 3. C. 375; Kriger V.G., Kalenskii A.V., Zvekov A.A., Zykov I.Yu., Nikitin A.P. // Thermophys. Aeromech. 2013. V. 20. N 3. P. 367. doi $10.1134 / \mathrm{S} 0869864313030153$

[27] Адуев Б.П., Нурмухаметов Д.Р., Звеков А.А., Никитин А.П., Каленский А.В. // Физика горения и взрыва. 2016. T. 52. № 6. C. 104; Aduev B.P., Nurmukhametov D.R., Zvekov A.A., Nikitin A.P., Kalenskii A.V. // Combust. Expl. Shock Waves. 2016. V. 52. N 6. P. 713. doi $10.1134 / \mathrm{S} 0010508216060113$ 